\title{
Innovatively exploring the constraints and challenges faced by malaria patients in the prevention and control of malaria-Nkhata Bay Malawi
}

\author{
John Damison Phiri ${ }^{*}$ \\ Thammasat University, Bangkok, Thailand
}

\section{Key Words: \\ Frequent stock outs of drugs \\ Preventable and curable \\ Reliable healthcare system \\ Unscientifically proven beliefs}

Received: 22 April 2016

Accepted: 10 May 2016

Published: 24 June 2016

\begin{abstract}
Malaria is a leading cause of morbidity and mortality in Malawi such that it accounts for over one-third of all outpatients annually. Further to this, it is a leading cause of hospital admission among children below the age of 5 years. It is responsible for about $40 \%$ of all hospitalization of this age group. Above all, malaria accounts for over $30 \%$ of in-hospital child death, and adults cannot work an average of 25 days in a year due to malaria. The incidence was currently standing at 484/1000 up from 458/1000 in 2009. This is despite several interventions and strategies being employed to try to reverse the trend. Qualitative research was used to explore the constraints and challenges faced by malaria patients in preventing and controlling malaria in Nkhata Bay Malawi. The confirmed malaria patients were put into four categories: Pregnant women, children below five years of age, adult females, and adult males, and in each category, a participant was identified through random sampling without replacement. Primary data from the participants were obtained through semi-structured interviews, and an analysis of the collected data was done using MS Excel. Ethical clearances were obtained from both Malawi and Thailand.
\end{abstract}

(C) 2016 The Author(s). Published by TAF Publishing.

\section{INTRODUCTION}

Malaria is an infectious disease caused by a parasite called plasmodium which is transmitted from an infected person to another through the bites of infected female anopheles mosquitoes. According to World Health Organization (WHO) [23], malaria is one of the top five killer diseases in the world, and second in Africa after HIV/AIDS [27]. The global health burden of malaria is enormous as 3.3 billion people in 97 countries and territories were at risk of being infected with it and developing the disease [27].

The recent statistics about malaria indicated that over 198 million cases were recorded globally in the year 2013 with 584000 of those cases translating into death. The report further indicated that of those deaths, $90 \%$ took place in Africa whilst $78 \%$ of those deaths in Africa were children below the age of 5 years [27]. The health burden due to malaria is much more enormous in Africa than in the other continents. This is so because over 100 million episodes of clinical malaria occur each year in the WHO African region. The actual figure is believed to be much higher than this as in most cases diagnosis of malaria is

\footnotetext{
${ }^{*}$ Corresponding author: John Damison Phiri

${ }^{\dagger}$ Email: johndphiri@yahoo.co.uk
} 
difficult to come up with because of imprecision in the clinical diagnosis and lack of microscopic confirmation [8], [17]. According to Connolly [4] of the available known five species of plasmodium that cause malaria (plasmodium falciparum, plasmodium vivax, plasmodium malariae, plasmodium ovale and plasmodium knowlesi) plasmodium falciparum and plasmodium vivax are mainly found in the sub Saharan Africa. Medicine for Malaria Ventures (http://www.mmv.org/malaria-medicines/five-species) indicated that plasmodium falciparum is the deadliest of all the species being responsible to be causing over $85 \%$ of the malaria cases in Africa and also being confirmed to be resistant to many antimalarial drugs such as chloroquine and sulfadoxine-pyrimethamine. Malaria is endemic in Malawi (Central Africa) and the entire population is at risk. Specifically, $97 \%$ of the population is at endemic risk whilst $3 \%$ is at epidemic risk [21].

According to National Statistical Office (NSO) [13] the population of Malawi was projected to be standing at 16.3 million people by end of 2015 comprising approximately $51 \%$ women and $49 \%$ men. Of this population $19 \%$ is children below the age of 5 years. This population is distributed in such a way that $86 \%$ live in rural areas whose dwelling houses comprises of poor quality houses constructed using mud, with grass thatched roofs [10]. The economic burden imposed by malaria on Malawi is so enormous as in the Malawi 2005-2010 Malaria Strategic Plan (MSP) titled 'Scaling up Malaria Control Interventions' it was defined as 'a disease of poverty-it affected the poorest people and kept them poor.' And in the 2011-2015 MSP titled 'Towards Universal Access' authorities admitted failure in meeting set targets which were set in the previous 2005-2010 MSP and were also contained in the 2000 Abuja Declaration and Roll Back Malaria (RBM).

\section{METHODOLOGY}

The study used primary data that was collected from the sampled participants through the use of semi-structured interviews which fall under qualitative method. Qualitative method emphasizes on understanding, interpretation, and observation in natural setting and in closeness to data [6]. The type of research approach for one to use depends on the kind of studies that will be conducted however it is argued that the beauty of using qualitative method in a research lies in the fact that the method takes into consideration the overall picture in a way quantitative method cannot [7].

A qualitative approach was deemed more appropriate in order to fulfill the purpose of this research as it was on exploring constraints and challenges faced by confirmed malaria positive patients in the prevention and control of malaria which meant that perceptions, beliefs, ideas and opinions from the patients were difficult to measure in a quantitative way.

The primary data was collected through the use of semi structured interviews which has an advantage that the respondent provide much more detailed information and above all the information so collected is provided in a more relaxed atmosphere [2]. According to McNamara [11] interviews are viewed as an important tool used to depict the story behind the interviewees' experiences and in this case the experiences on the constraints and challenges faced by the confirmed malaria positive patients.

\section{Population and Sample}

The target population for this study comprised all confirmed malaria positive patients to Nkhata Bay District Hospital. The total number of participants to this study were 74 (seventy four) and were put in four categories namely: pregnant women, children under 5 years, adult females and adult male as outlined in Table 1. Patton [14] defined sampling as 
TABLE 1. Composition of participants

\begin{tabular}{ll}
\hline \hline Category & Number/Category \\
\hline Pregnant women & 12 \\
Children below 5 years & 12 \\
Adult female & 25 \\
Adult male & 25 \\
\hline \hline
\end{tabular}

the selection of individuals, units, and or settings to be studied and in the case of this research sampling therefore referred to the selection of confirmed malaria positive patients to participate in the study. Participants in all the four categories were identified through random sampling without replacement such that after the first confirmed malaria positive patient was taken, then the next one was the one coming after 5 confirmed malaria positive patients. The confirmed malaria positive patients were purposefully sampled. In terms of purposive sampling according to Denzin et al. [5] and Merriam [12] refers to the selection of interviewees' based on an important characteristic understudy such as where one lives (rural or urban) position in society and so on. In this case therefore all the sampled participants were confirmed malaria positive patients adult (male and female), pregnant women and children. All these had characteristics relevant to the study which was on constraints and challenges faced by the malaria patients. Since the primary data was collected from confirmed malaria positive patients meant that their practical knowledge and experience supported the consistency of the data and that the data so collected from them about their experiences was valid and reliable.

\section{RESULTS}

The results of the study have been divided into various categories such as preventive, curative, Information Education and Communication (IEC) and Healthcare systems. Each of these said categories have their own constraints and challenges which the malaria patients did experience.

\section{Prevention}

As the old saying goes that prevention is better than cure in the sense there is a lot of economic saving if any disease is prevented rather than one contract it and then gets cured from it. From the immense literature available, malaria is an infectious disease caused by a plasmodium parasite and that it is both preventable and curable. The study found that the participants had knowledge of what malaria was and how it was transmitted from one infected person to another.

From the table on knowledge of how malaria was transmitted, the results showed that there was $100 \%$ knowledge of the disease in three categories namely: pregnant women, children under the age of 5 years (mothers/guardians) and in the adult male categories. However, in the adult female category of the sampled malaria positive patients, knowledge of the disease was at $96 \%$ whilst $4 \%$ did not know how malaria was transmitted.

In terms of the overall population sample thus combining all the categories, the study revealed that $98.6 \%$ knew how malaria was transmitted while on the other hand $1.4 \%$ did not know how malaria was transmitted from one person to another. Of the available preventive interventions, the study found that they were using Indoor Residual Spray (IRS), Insecticide Treated Bed Nets (ITNs), burning of dry cow dung and intermittent preventive treatment of malaria in pregnancy with Sulfadoxine-Pyrimethamine (IPTp-SP) in the case 
TABLE 2 . Knowledge on how malaria was transmitted

\begin{tabular}{llll}
\hline \hline Category & \multicolumn{2}{c}{ Knowledge } & Total \\
\hline & Knew & Didn't Know & \\
\hline Pregnant women & 12 & 0 & 12 \\
& $100 \%$ & $0 \%$ & $100 \%$ \\
$U_{5}$ Children & 12 & 0 & 12 \\
(Mothers/Guardians) & $100 \%$ & $0 \%$ & $100 \%$ \\
Adult Female & 25 & 1 & 25 \\
& $96 \%$ & $4 \%$ & $100 \%$ \\
Adult Male & 25 & 0 & 25 \\
& $100 \%$ & $0 \%$ & $100 \%$ \\
Total & $73(98.6 \%)$ & $1(1.4 \%)$ & \\
\hline \hline
\end{tabular}

of pregnant women. However, there were variations in knowledge among the participants on each intervention as well as preference for using the chosen intervention. Various reasons were actually learnt as to why they preferred the intervention they were using over the other available interventions.

\section{Knowledge of and Practicing of Burning Dry Cow Dung}

The study found that $85 \%$ of the sampled population knew that burning of dry cow dung in the bedroom as a traditional method would actually drive out mosquitoes whilst $15 \%$ was ignorant of this traditional method. However despite $85 \%$ of the sampled population having knowledge of this dry cow dung burning only $7 \%$ actually practiced the burning of this dry cow dung whilst $16 \%$ only believed in the traditional method without actually practicing it.

Despite having heard of it, $77 \%$ did not believe in the burning of dry cow dung as a means of driving out mosquitoes. As for the $7 \%$ which practiced this method did so because it was felt to be economical as they could not part up with any money in order to buy the dry cow dung.

\section{Knowledge of ITNs}

The ITNs if properly used act as a physical barrier between the persons sleeping under them and the mosquitoes thereby reducing the number of bites from the infective mosquitoes [9]. The study revealed the knowledge the sampled malaria positive patients had on ITNs. It was revealed that the whole sample thus $100 \%$ knew what ITNs were all about, what they were meant to be used for and how they were supposed to be used. However there were variations in ownership and usage of those ITNs.

\section{Ownership and Usage of Owned ITNs}

Of the four categories, pregnant women and children under 5 years of age, adult female and adult male, only two categories namely pregnant women and children below the age of 5 years were the beneficiaries of free ITNs issued on to them in all government health facilities and Christian Health Association of Malawi (CHAM) health facilities throughout the country. These categories were regarded as vulnerable as their immunity was low. In the case of a pregnant woman her immunity gets lowered immediately she becomes pregnant whilst for the children below the age of 5 years their immunity is not yet fully developed and thus why they were known to be vulnerable. The adult female and adult males had to buy ITNs from the local shops. 
TABLE 3. Ownership and usage of owned ITNs

\begin{tabular}{|c|c|c|c|c|}
\hline \multirow[t]{2}{*}{ Category } & \multicolumn{2}{|c|}{ Ownership } & \multicolumn{2}{|c|}{ Usage } \\
\hline & Owned ITN & Didn't Own ITN & Used ITN & Didn't Use ITN \\
\hline \multirow[t]{2}{*}{ Pregnant women } & $12100 \%$ & 0 & $433.3 \%$ & $866.7 \%$ \\
\hline & & $0 \%$ & & \\
\hline \multirow[t]{2}{*}{$U_{5}$} & children & $12100 \%$ & $0758.3 \%$ & $541.7 \%$ \\
\hline & & $0 \%$ & & \\
\hline \multirow[t]{2}{*}{ Adult female } & $1456 \%$ & $1144 \%$ & $642.9 \%$ & $857.1 \%$ \\
\hline & $56 \%$ & & & \\
\hline \multirow[t]{2}{*}{ Adult male } & $832 \%$ & 17 68\% & 8 & 0 \\
\hline & $32 \%$ & & $100 \%$ & $0 \%$ \\
\hline Total & $4662.2 \%$ & $2837.8 \%$ & $2558.6 \%$ & $2141.4 \%$ \\
\hline
\end{tabular}

It came out that the study revealed that in the pregnant women and children less than 5 years categories ownership was at $100 \%$ while in the adult female category ownership stood at $56 \%$ with $44 \%$ not owning any ITN and in the adult male category ownership of ITNs stood at $32 \%$ with $68 \%$ not owning any ITN. However, ownership in terms of the whole sample was at $62.2 \%$ with $37.8 \%$ not owning any ITN. In terms of usage of the owned ITNs, the study found that: Out of the $100 \%$ which owned ITNs in the pregnant women category only $33.3 \%$ were using them with $66.7 \%$ not putting the owned ITNs into use whilst in the children less than 5 years, out of the $100 \%$ which owned ITNs, only $58.3 \%$ were using them with $41.7 \%$ not using them.

On the other hand out of the $56 \%$ that owned ITNs in the adult female category only $42.9 \%$ were putting them into use with $57.1 \%$ not using them. As in the adult male category, out of the $32 \%$ that owned ITNs, the whole $100 \%$ was using the ITNs. In terms of the whole sample comprising all the four categories, usage of the owned ITNs was at 58.6\% with $41.4 \%$ not using them. Some of the reasons that the study found for non- usage of the ITNs in the case of pregnant women and children less than 5 years, were that husbands forced them to sell the ITNs they had received from the health facilities.

The aim was to find money to solve immediate pressing problems. Non-usage of ITNs across the whole sampled patients were that the bed nets were being diverted from instead of sleeping under them to be used for fishing, drying fish, and others covering their small vegetable gardens in their homes protecting the vegetables from chicken and other domestic animals. The study found that husbands used force on those who were willing to use the ITNs in sleeping under them besides diverting ITNs based on the fact that medical services in the country were fully subsidized. Since the health services were rendered for free to the people, it was generally felt that there was no need to sleep under ITNs because if they would fall sick from malaria or any disease they would simply go to the hospital and get treatment which was free on their part. This can be termed as a free health service delivery syndrome. The study also found unscientific proven beliefs to be preventing the patients from using ITNs and these were that if a pregnant woman slept under an ITN during her pregnancy, then the baby to be born would be born with some albinism. Also that if a couple slept under an ITN then the woman would fail to conceive else if by chance she conceived then the baby will be born with albinism.

To a child who was made to sleep under an ITN would not grow up as a healthy child if at all that child will grow. Further to this the study found that sleeping under an ITN was felt like being in a grave and again traditionally it was not acceptable for one to sleep in two houses the same night referring to an ITN as a house erected inside another house. 


\section{Misconceptions of Interventions}

World Health Organization [26] recommends intermittent preventive treatment of malaria in pregnancy with an efficacious drug which currently is sulfadoxine-pyrimethamine (IPTpSP). IPTp involves an administration to all pregnant women at least 2 doses of SP during the second and third trimesters of pregnancy irrespective of presence of signs of malaria infections. However, besides the IPTp-SP, WHO further recommends pregnant women to be sleeping under ITNs [22] and in areas of high plasmodium falciparum transmission the administration of IPTp-SP should start as early as possible in the second trimester [27] and at each scheduled ANC visit up to delivery time without any safety concerns provided a gap of one month elapsesin between administrations of SP [15]. The study found that $42 \%$ of the sampled malaria positive pregnant patient mistook SP that was given to them as a stand-alone intervention and as such once given they would not consider using other preventive interventions. On the other hand $58 \%$ took SP not as a complete stand-alone intervention. The study further found that the administered SP was swallowed in the presence of a health worker.

\section{Prevention Using Indoor Residual Spray(IRS)}

Vector control interventions such as ITNs and IRS are the key malaria control tools [24], [25]. IRS can be defined as the occasional spraying of inside house walls with a persistent insecticide to reduce mosquito's lifespan and density hence a reduction in the transmission of malaria. However the study found that only $70 \%$ of the sampled patients had their houses sprayed during the time the IRS was being carried out in the district. The main reason found for not having sprayed all the houses being that those whose houses were not sprayed were not comfortable with the strong smell produced by the pesticides used. On the other hand the team conducting the IRS had under estimated the required amount of pesticides.

\section{Preference of Preventive Interventions}

The study found that $12 \%$ of the sampled malaria patients preferred to be protecting themselves against mosquitoes through the use of IRS whilst $62 \%$ preferred the use of ITNs and $19 \%$ opted for both IRS and ITNs, starting with IRS and then followed by ITNs while the remaining $7 \%$ preferred the burning of dry cow dung. Various reasons as to why the patients preferred a particular intervention over the others were found to be that: ITNs were easy to use and could be easily carried in event that patients for whatever reason were moving from one place to another and eventually night stopping there. An ITN could be mounted at a place where the patient intended to spend a night hence protecting themselves from mosquito bites. However, $12 \%$ who opted for IRS argued that despite the strong smell produced by the pesticides used in IRS, protection was accorded to everyone who was sleeping in the house that has been sprayed and could not be diverted once sprayed which was an indirect reference to what was happening with ITNs. Considering the number of people who sleep under one roof due to the extended family system, it was difficult to have an ITN for each one setup hence IRS guaranteed protection for everyone. Those who preferred the using of both IRS and ITNs starting with IRS then followed up by ITNs argued that once IRS was done, not all mosquitoes would be killed as the mosquitoes themselves were not stationary. As the pesticides used in the IRS moved towards the end of their effectiveness, mosquitoes would still find their way into the sprayed houses hence the using of ITNs was a backup to the IRS. On the other hand due to economic hardships others preferred burning of dry cow dung which was sourced free of charge. 


\section{Availability of Openings on Their Dwelling Houses}

Anopheles mosquitoes have developed behaviors to more efficiently feed on humans and would fly upwards upon reaching a wall while other mosquitoes would fly sideways. Flying upwards upon reaching a wall helps the mosquitoes find openings such as windows, cracks around doors and open eaves [1]. So mosquitoes would use any opening available on any part of the house in order to go through. It was found that $93 \%$ of the participants had their houses with other openings on the walls other than the windows and doors. It was further found that $82 \%$ of the owners of those dwelling houses with openings at times try to screen those openings however the materials used or method employed were not all that effective to prevent mosquitoes from passing through whilst $18 \%$ did not attempt to screen the openings. Screening may reduce malaria transmission as was experienced in British Barracks in Amritsar in India who after screening in 1925 reduced the incidence of malaria from $613 / 1000$ to $48 / 1000$ in 1927 [1]. The study revealed that the nature of the houses of the participants and how they were constructed necessitated the availability of those openings and it was just difficult for them to screen those openings. Besides, poverty was making it difficult for them to acquire appropriate and durable screening materials such as wire mesh.

\section{Source of Water for Domestic Use and Approximate Distances from the Source}

Just like all other mosquitoes, anopheles mosquitoes go through four stages in their lifecycle and these are eggs, larva, pupa and adult. The first three stages are aquatic and according to Centre for Disease Control and Prevention [3] prefer slow moving or still water in which they can stay close to the surface to ease breathing. Unlike the other types of mosquitoes, anopheles requires clean water for the development of their larva [3]. Upon reaching adult anopheles mosquitoes are known to have a flight range of usually (radius of $1.5 \mathrm{~km}$ ) 3km from their breeding sites [20] implying that if houses were located less than this distance from mosquito's breeding sites then the mosquitoes can easily reach those houses.

The study found that $50 \%$ of the participants used borehole as their source of water while $8 \%$ used open well and $42 \%$ used tap water. Of these participants the study further found that $23 \%$ had traces of standing water from their various sources of water with $77 \%$ claiming that their areas had no traces of standing water. However, mosquitoes do not need big pools of standing water in order to complete their lifecycle. In terms of distances to the sources of water for domestic use, the study revealed that only $5 \%$ of the sampled participants were covering a distance of more than $1.5 \mathrm{~km}$ which was a clear indication that the rest of the participants had their houses within the flight range of mosquitoes from their breeding sites.

\section{Frequency Patients Suffer from Malaria}

Despite 98.6\% having knowledge on how malaria is transmitted and all the preventive interventions at their disposal, the study found that $18 \%$ fell sick from malaria every month, $38 \%$ fell sick from malaria at least once in every 3 months and $26 \%$ suffered from malaria at least half yearly. $13 \%$ of the participants suffered from malaria at least once a year and finally $5 \%$ suffered from malaria after every year. There were variations in terms of time patients would consider accessing medical help once symptoms of malaria were noted in them. WHO recommends that effective treatment should commence within 24 hours of the onset of symptoms [26]. However it was found that only $28 \%$ complied to the WHO's recommendation whilst the other $72 \%$ did not. Others even took more than 1 week before 
accessing medical help. Among the reasons the study found to be behind this non- compliance was the long distances the patients had to travel to health facility and associated high transportation cost coupled with frequent stock outs of drugs at the health facility.

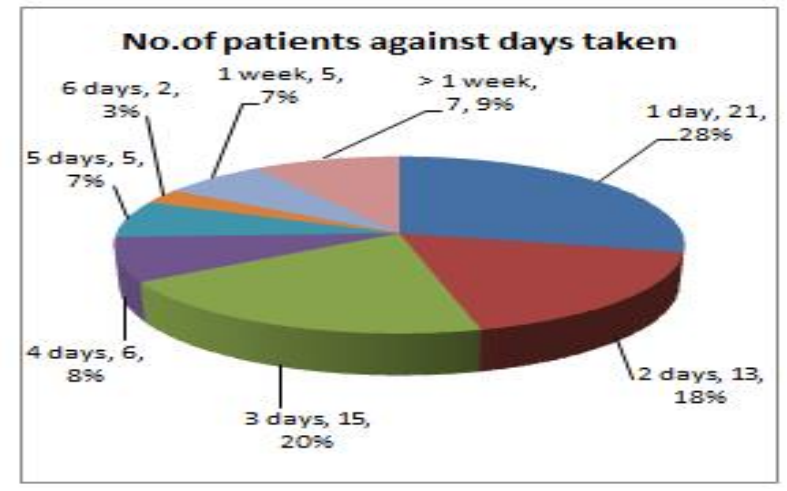

FIGURE 1. Time taken before seeking medical help

\section{Healthcare System}

World Health Organization [28] recommends that only those that have been confirmed malaria positive either through RDT or microscopically should be the ones to be prescribed anti-malarial drugs. In the malaria endemic countries like Malawi, malaria's clinical symptoms are shared with other disease, hence diagnosis based on clinical signs and symptoms alone tended to result in most cases over diagnosis of malaria, leading to wastage of resources and failure to treat the true cause of the patients' illness as almost all fever cases were treated for malaria [16], [19].

However, the study found that all the facility's microscopes were non-functional and that supply of drugs and RDTs was erratic. Even if the RDTs were available some of the patients were not in favor of giving blood samples for RDTs on the basis that they as patient were already weak and taking blood from such patients might make them weaker whilst those who accepted did so with the idea of wanting the health worker to establish their real problem.

\section{CONCLUSION}

The study has revealed some of the factors that contribute to the patients be failing to prevent and control the malaria disease. Some of the noted factors were:

- Patients failing to use ITNs because of unscientifically proven beliefs of what might come out of them if the slept under those ITNs.

- Diverting intervention materials such as ITNs and using them for fishing, drying up fish and covering vegetable gardens.

- Preference of unscientifically proven intervention such as burning of dry cow dung over those interventions that have been scientifically proven and whose usage helped some parts of the world to eradicate malaria or reduce it to a level of no public significance.

- Lack of clear communication resulting into pregnant women mistaking IPTp-SP as a complete stand-alone intervention which was against the WHO recommendations.

- The non-screening of the available openings on the walls of the dwelling houses enabled mosquitos' free entry into the various houses. Again the houses were located at distances within the flight range of mosquitoes from their breeding sites.

- Long distances to be covered by patients to health facility hampered efforts of seeking medical help within the WHO's 24 hours recommendations of the onset of the signs and 
symptoms of the disease. Further to this the persistent stock outs of prescribed drugs demotivated the patients from going to the hospital.

- An abuse of the fully subsidized medical services by the patients to the effect that it was felt there was no need to take personal responsibility over preventing themselves against malaria because if they fall sick from malaria or any disease they would go to the hospital and get free treatment.

\section{RECOMMENDATIONS}

If the constraints and challenges were to be minimized leading to an eventual elimination of malaria then there has to be some innovations. Schermerhorn [18] defined innovation as coming up with new ideas and putting them into practice. In this regard the author is suggesting the following process innovations:

1. The ITNs should be made available to all people irrespective of their level of immunity and engage the husbands on the benefits of using ITNs as the study has revealed $100 \%$ usage of owned ITNs in the adult male category. Also engage the local leaders on how best the unscientifically proven beliefs can be removed from the peoples' minds.

2. Change the distribution pattern of the ITNs from health facility based to community based.

3. Communities should enact by-laws which will have to regulate usage of preventive materials.

4. Introduce volunteers to monitor usage of ITNs in the community and that those diverting or not using the ITNs should be disciplined based on the by-laws the communities can come up with.

5. Sensitize and motivate the communities to cover all the minute ponds in their areas and make their surroundings always clean and clear and also to screen any openings on their houses.

6. Though the health services are fully subsidized, introduce some fee so that there is sense of ownership in the treatment process.

7. Bring health services close to people by introducing reliable scheduled mobile clinics not only for malaria but for all the common diseases. The health authorities should ensure that their stocks of pharmaceutical products are backed up by their demand with of course a mark-up margin.

8. Authorities should strive to establish a reliable healthcare system with no stock outs of essential products.

\section{REFERENCES}

1. Anderson L, Simpson D, Stephens M. Effective malaria control through durable housing improvements. [White paper no. 1]. Global Programs Department, Habit for Humanity, Atlanta, GA; 2014.

2. Boyce C, Neale P. Conducting in-depth interviews: A guide for designing and conducting in-depth interviews for evaluation input. Pathfinder International, Watertown, MA; 2006.

3. Center for Disease Control and Prevention (CDC). Malaria general information-global health-division of parasitic disease and malaria. U.S Department of Health and Human Services, Atlanta, GA; 2012.

4. Connolly MA. Communicable disease control in emergencies: A field manual. World Health Organization, Geneva, SW; 2005.

5. Denzin NK, Lincoln YK. Handbook of qualitative research. Newbury Park, CA: Sage Publication; 1984. 
6. Ghauri P, Grønhaug K. Research methods in business studies: A practical guide. Harlow, UK: Financial Times and Prentice Hall; 2002.

7. Gunnarson R. Forskning metoder validitet och reliabilitet. 2002. Available from: http://goo.gl/RvZCw3

8. Greenwood B. Malaria mortality and morbidity in Africa. Bulletin of the World Health Organization. 1999; 77(8): 617-618.

9. Lindsay S, Gibson ME. Bednets revisited-Old idea, new angle. Parasitology Today. 1988; 4(10): 270-272. D0I: 10.1016/0169-4758(

10. Malawi Government. Malawi state of environment and outlook report. Environment for sustainable economic growth. Environmental Affairs Department, Lilongwe, MW; 2010.

11. McNamara C. General guidelines for conducting interviews. 1999. Available from: http://goo.gl/D66DBW

12. Merriam SB. Qualitative research and case study: Application in education. San-Francisco, SA: Jossey-Bass Publication; 1998.

13. National Statistical Office (NSO). Population and housing census. National Statistical Office, Zomba, MW; 2008.

14. Patton MQ. Qualitative evaluation and research methods. Newbury Park, CA: Sage Publications; 2001.

15. Peters PJ, Thigpen MC, Parise ME, Newman RD. Safety and toxicity of sulfadoxine/pyrimethamine. Drug Safety. 2007; 30(6): 481-501. DOI: 10.1016/0169-4758(88)90017-8

16. Reyburn H, Mbatia R, Drakeley C, Carneiro I, Mwakasungula, E, Mwerinde O, Greenwood BM. Overdiagnosis of malaria in patients with severe febrile illness in Tanzania: A prospective study. BMJ. 2004; 329(7476): 1-6.

DOI: $10.1136 / \mathrm{bmj} .38251 .658229 .55$

17. Samba E. Preface: The malaria burden and Africa. The American Journal of Tropical Medicine and Hygiene. 2001.

18. Schermerhorn JR. Introduction to management. New Jersey, NJ: John Wiley \& Sons; 2013.

19. Thiam S, Thior M, Faye B, Ndiop M, Diouf ML, Diouf MB, Lee E. Major reduction in anti-malarial drug consumption in Senegal after nation-wide introduction of malaria rapid diagnostic tests. Plos One. 2011; 6(4): e18419. DOI: 10.1371/journal.pone.0018419

20. Walker K. A review of control methods for african malaria vectors. Activity report 108 environmental health project office of health infectious diseases and nutrition. Bureau for Global Health US Agency for International Development, Washington, DC., WA; 2002.

21. Wallon ME, Roman W, Brieger RBA. Malaria in pregnancy case study: Zambia's successes and remaining challenges for malaria in pregnancy programming. Maternal and Child Health Integrated Program, Washington DC., WA; 2010.

22. World Health Organization/Africa region (WHO/Afro). A policy framework for malaria prevention and control during pregnancy in the African region. World Health Organization, Brazzaville, CG; 2004.

23. World Health Organization (WHO). A strategic framework for malaria prevention and control during pregnancy in the African Region. World Health Organization Regional Office for Africa, Brazzaville, CG; 2004.

24. World Health Organization (WHO). World Health Organization gives indoor use of DDT a clean bill of health for controlling malaria. World Health Organization, Geneva, SW; 2006.

25. World Health Organization (WHO). Global malaria programme insecticide treated mosquito nets. $\boldsymbol{A}$ WHO position statement. Geneva, SW: World Health Organization; 2007.

26. World Health Organization (WHO). Guidelines for the treatment of malaria. Geneva, SW: WHO Library Cataloguing-inPublication Data; 2010.

27. World Health Organization (WHO). World Health Organization policy brief for the implementation Fof intermittent preventive treatment of malaria in pregnancy using Sulfadoxine-Pyrimethamine (IPTp-SP). Geneva, SW: World Health Organization; 2013.

28. World Health Organization (WHO). World Malaria Report. Geneva, SW: WHO Library Cataloguing-in-Publication Data; 2014.

— This article does not have any appendix. - 